Referencia para citar este artículo: Cifuentes-Muñoz, A., \& Rojas-Jara, C. (2018). La fotografía como medio narrativo para la co-construcción de identidades alternativas en contexto de abuso de drogas. Revista Latinoamericana de Ciencias Sociales, Niñez y Juventud, 16(1), 89-100. doi:10.11600/1692715x.16104

\title{
La fotografía como medio narrativo para la co-construcción de identidades alternativas en contexto de abuso de drogas*
}

\author{
Alejandro Cifuentes-Muñoz ** \\ Universidad de Talca, Chile. \\ CLAUDIO ROJAS-JARA *** \\ Universidad Católica del Maule, Chile.
}

Artículo recibido en septiembre 6 de 2016; artículo aceptado en noviembre 2 de 2016 (Eds.)

- Resumen (descriptivo): en este artículo presentamos una reflexión teórica, enmarcada epistemológicamente desde el construccionismo social, sobre cómo los retratos fotográficos pueden configurarse como un medio narrativo que facilita la co-construcción de identidades descentradas y alternativas a la identidad dominante de drogadicto. Abordamos la red teórica que otorga sustento a tal reflexión y posteriormente describimos una aplicación práctica de esta junto a un grupo de jóvenes en contexto de abuso de drogas. Concluimos que la fotografia se configura como un medio narrativo para la utilización terapéutica en la medida en que es posible atribuirle significados a través del lenguaje, emergiendo como un medio respetuoso, innovador y pragmático que pudiera enriquecer los tratamientos convencionales de drogas, en lo que respecta a la promoción de identidades alternativas.

Palabras clave: drogas, fotografía, identidad (Tesauros de Ciencias Sociales de la Unesco)

Palabras clave autores: construccionismo social, narrativa.

\section{Photography as a Narrative Medium for the Co-Construction of Alternative Identities in the Context of Drug Abuse}

- Abstract (descriptive): In this article we present a theoretical reflection, framed epistemologically from social constructionism, on how photographic portraits can be configured as a narrative medium that facilitates the co-construction of decentred and alternative identities to the dominant identity of drug addict. We approach the theoretical network that supports this reflection and later we describe a practical application of this with a group of young people in the context of drug abuse. We conclude that photography is configured as a narrative medium for therapeutic use to the extent that as it is possible to give meanings to it through language, emerging as a respectful, innovative and pragmatic medium that could enrich conventional drug treatments, related to the promotion of alternative identities.

\footnotetext{
* Este artículo de revisión de tema, que pertenece al área de las ciencias sociales y a la sub área de psicología, es fruto de una ponencia presentada, en modalidad de mesa temática sobre los usos terapéuticos de la fotografía, en el $12^{\circ}$ congreso chileno de investigación en psicoterapia y $14^{\circ}$ congreso chileno de psicoterapia Reñaca, en agosto de 2016.

** Psicólogo, Licenciado en psicología mención Psicología de la Salud Universidad Católica del Maule, Chile. Magíster en Psicología Clínica mención Psicoterapia Constructivista y Construccionista Universidad de Valparaíso, Chile. Doctorando en Ciencias Humanas Universidad de Talca, Chile. Orcid: 0000-0003-1767-5517. Correo electrónico: acifuentes@utalca.cl

*** Psicólogo, Licenciado en Psicología, mención Psicología de la Salud, Universidad Católica del Maule. Magíster en Drogodependencias, Universidad Central de Chile. Académico Departamento de Psicología, Facultad de Ciencias de la Salud, Universidad Católica del Maule, Chile. Orcid: 0000-0002-1698-6949. Correo electrónico: crojasj@ucm.cl
} 
Key words: Drugs, photography, identity (Social Sciences Unesco Thesaurus). Authors key words: Social constructionism, narrative.

\section{A fotografia como um meio narrativo para co- construção de identidades alternativas no contexto do abuso de drogas}

- Resumo (descritivo): neste artigo, apresentamos uma reflexão teórica, enquadrada epistemologicamente pelo construcionismo social, sobre a forma como os retratos fotográficos podem ser configurados como um meio narrativo que facilita a co-construção de identidades descentralizadas e alternativas à identidade dominante do toxicodependente. Abordamos a rede teórica que apoia essa reflexão e depois descrevemos uma aplicação prática deste, juntamente com um grupo de jovens no contexto do abuso de drogas. Concluímos que a fotografia é configurada como um meio narrativo para uso terapêutico na medida em que é possível atribuir significados a ela através da linguagem, emergindo assim como um meio respeitoso, inovador e pragmático que poderia enriquecer os tratamentos de drogas convencionais, no que se refere à promoção de identidades alternativas.

Palavras-chave: drogas, fotografia, identidade (Thesaurus de Ciências Sociais da Unesco).

Palavras-chave autores: construcionismo social, narrativa.

-1. Introducción. -2. Construccionismo social, narrativa e identidad. -3. Identidad dominante de drogadicto. -4. Fotografía terapéutica, fotografía social y retrato. -5 . ¿De qué manera la fotografía se configura como un medio narrativo? -6. De la reflexión teórica a la pragmática: Una experiencia de trabajo. -7. Reflexiones finales. -Lista de referencias.

\section{Introducción}

Podríamos comenzar este escrito con datos epidemiológicos y estadísticos respecto al consumo de drogas en el mundo o en Chile; sin embargo, no buscamos aquí describir cuantitativamente el fenómeno de las drogas sino, por el contrario, promover la reflexión crítica sobre él, sobre la manera en que lo entendemos y sobre las prácticas con las que lo abordamos.

El consumo de drogas es comprendido y enfrentado mayoritariamente mediante dos modelos dominantes y hegemónicos a nivel mundial: el modelo criminalizador y el modelo de enfermedad. El primero centrado en el castigo respecto a estándares morales y el segundo centrado en la patología y criterios diagnósticos. Ambos transgreden de igual forma los derechos humanos de las personas (Oda, 2015). Chile no es la excepción en la utilización de tales modelos; basta decir que el Servicio Nacional de Drogas y Alcohol (Senda) se encuentra inserto en el Ministerio del Interior, el cual se encarga de la seguridad pública. Por otro lado, y en referencia al modelo de enfermedad, los diagnósticos de abuso o de dependencia de drogas y tratamientos estandarizados para “curar esta patología” mediante terapias basadas en la evidencia, son pan de cada día en los centros de tratamiento a lo largo del país. Lo perturbador de tales concepciones es que están centradas en variables intrapsíquicas y en la universalidad del dolor humano, dejando de lado lo más importante a nuestra consideración: las historias donde se aloja un problema y el derecho de cada persona a contar tal historia en sus propios términos, donde no sea el ser humano el que se adapte a un sistema castigador o patologizador, sino que sea el profesional el que pueda adaptarse a la red de significados de la persona, protagonista de su historia y de su relación, - -ya sea esta problemática o no según su propia definición - respecto al consumo de drogas. Tal tarea no es fácil de llevar a cabo, ya que la sociedad que habitamos y construimos está hegemónicamente diseñada para funcionar así y nosotros como profesionales estamos educados para promover su perpetuación. El primer paso para un cambio, en nuestra opinión, tendría necesaria relación con la pregunta sobre el operar epistemológico mediante el 
cual nos cuestionemos de qué manera hemos comprendido al ser humano y a la configuración de su realidad, para posteriormente llevar a cabo abordajes prácticos consecuentes con tal respuesta.

En consecuencia, el horizonte epistemológico de este artículo de reflexión converge sobre una mirada construccionista social, donde consideramos que el ser humano construye su realidad a través de elementos lingüísticos. Desarrollamos la tesis sobre cómo el uso del retrato fotográfico puede configurarse como un medio narrativo, facilitador $\mathrm{y}$ promotor de nuevas identidades en jóvenes drogodependientes. Luego de dar cuenta de los soportes teóricos y epistemológicos de tal reflexión, presentamos un diálogo de esta tesis teórica con la práctica, donde a modo de ejemplo, describimos una experiencia de trabajo terapéutico que se basa en la reflexión que desarrollamos junto a un grupo de jóvenes pertenecientes a un centro de rehabilitación de drogas en Talca, Chile.

\section{Construccionismo social, narrativa e identidad}

En palabras de Gergen (1985) diríamos que el construccionismo social pretende mostrar la forma en que las personas llegamos a describir, explicar o dar cuenta del mundo que habitamos; ello se configura en una postura epistemológica posmoderna, que busca entender la naturaleza de la experiencia del ser humano y la manera en que este construye su realidad. Dentro de esta postura, aceptamos que la realidad es socialmente construida a través del lenguaje, asumiendo que no es posible tener un conocimiento objetivo y directo del mundo, sino solo acceder a él con base en la interpretación o significado de nuestra experiencia, cuestionando constructos como la verdad, lo real y la objetividad. Las conceptualizaciones, descripciones y significados que hacemos del mundo se construyen a través del lenguaje como producto de las relaciones entre las personas y sus interacciones lingüísticas. Múltiples pueden ser los significados que atribuimos a una experiencia; no obstante, no siempre somos completamente libres de otorgar el significado que deseamos, sino que estos están conjugados al alero de discursos dominantes que inciden en la significación que cada uno de nosotros le otorga a un hecho o experiencia. Así, entendemos que la construcción social del significado tiene directa relación con el poder acerca de cómo se deciden ciertos significados y no otros; lo anterior al pie de la hegemonía establecida por unos con poder que influencian a otros sin poder, impactando en las comunidades lingüísticas $\mathrm{y}$, por ende, en el significado (Vilches, 2007). Sin embargo, es la propia construcción de significados compartidos la que otorga una posibilidad y alternativa para salir de los problemas o sufrimientos colectivos o individuales, respectivamente. En esta línea, Sánchez-Jiménez (2017) plantea que “en este marco epistemológico, la creación de significado, la experiencia y el conocimiento son procesos constructivos con capacidad potencial de transformar las pautas de relación social entre las personas” (p. 1182).

Una narrativa, nos dice Sluzki (1996), es un sistema de significados compartidos constituido por actores o personajes que incluye un guion, un escenario y un sentido. De tal forma, para comprender qué es la narrativa y su relación con la construcción de identidad, es necesario reflexionar sobre el concepto de self o sí mismo que conlleva la narración. Goolishian y Anderson (2005) refieren al self como narrador, entendiendo que el sí mismo es el resultado del proceso humano de producción de significado por medio de la acción del lenguaje. En palabras concretas, el sí mismo es la manera en que nos contamos historias respecto a qué somos y quiénes somos mutuamente, tanto con nosotros mismos como con otros, incluyendo las historias que otros relatan de nosotros mismos. De tal forma, la narrativa, como constitutiva del self, es una manera de contar nuestra propia individualidad de forma cambiante; no fija en un espacio ni en un tiempo, dando cabida a la construcción de nuestra identidad. Anderson (1997) señala que la "narrativa es la forma en que organizamos, explicamos, damos sentido 
y comprendemos, es decir, damos estructura y coherencia a las circunstancias y sucesos de nuestra vida, los fragmentos de nuestras experiencias e identidades propias para y con nosotros y otros” (p. 277).

\section{Identidad dominante de drogadicto}

La posibilidad de transitar de una identidad a otra se encuentra bajo el yugo del contexto social. Molina, Raimundi y Giménez (2017), respecto a la identidad, establecen que "los posibles sí mismos son una construcción social. Un individuo puede crear una variedad de sí mismos potenciales pero éstos derivan de las categorías salientes en su contexto sociocultural e histórico” (p. 457). Asimismo, White y Epston (1993) refieren que la identidad es una construcción colectiva en donde incide el contexto sociocultural e histórico particular de cada persona. Por tanto, las relaciones de poder generan metanarrativas normalizadoras que suscitan descripciones pobres de identidad en las personas, generando identidades dominantes centradas en problemas. La propuesta social hegemónica centrada en un discurso dominante de enfermedad o castigo en el tratamiento de drogas, por ejemplo, condiciona y limita las historias que pueden ser narradas por las personas que participan en los tratamientos, restringiendo la posibilidad de contarse historias distintas, descentradas de aquello que genera dolor y que definen su identidad como drogadicto, limitando las identidades alternativas.

Hemos referido que las narrativas dan emergencia a la identidad, por ende, para entender la construcción de identidad de drogadicto, debemos conocer primero cuáles son las narrativas asociadas a esta, tanto por las propias personas con relaciones problemáticas con la droga, como por otros, incluidos los terapeutas. Narrativas centradas en "ya no sé qué hacer", "la droga me controla", "siento que es lo único que me hace feliz", "no puedo sin ella", "me atrapa", "no tengo remedio", "me merezco lo que me pasa”, "soy el drogadicto de la familia", entre otras, se configuran como elementos constitutivos en la identidad de un joven, siendo estas narrativas dominantes, centradas en una identidad de drogadicto, las que limitan las posibilidades de narración de historias alternativas que no estén alojadas en el sufrimiento y que permitan la construcción de una identidad alternativa y descentrada de este.

Como pudimos revisar, las identidades no solo se construyen respecto a las historias que las propias personas se cuentan, sino también a lo que otros cuentan de ellos. Es acá donde Rojas (2015) refiere cómo muchas veces los propios terapeutas pasamos a ser perpetuadores de modelos dominantes, cuando asignamos significados a una persona que mantiene problemas con las drogas como, por ejemplo: "emocionalmente inestables, poco tolerantes a las frustraciones, impulsivos, débiles, agresivos, irresponsables, con baja autoestima, manipuladores o mentirosos” (p. 26), considerando tales características como constitutivas de su identidad. Es más, tanto la sociedad en general como las propias familias de las personas en situación de adicción, construyen redes de significados asociadas al estigma del consumidor de drogas, como bien lo ejemplifica Chacón (2015), en su capítulo "Estigma y drogas: 'cuidado con los volados de la esquina'”. Así, encontramos narrativas que forman parte de las personas en situación de adicción, así como en sus familias, en la sociedad en general e incluso en los propios terapeutas, tributando tales historias a la construcción de la identidad de drogadicto.

Recapitulemos en función de lograr una mejor comprensión del proceso de construcción de identidad a través de narrativas: cada distinción lingüística que realiza un observador da lugar a descripciones y explicaciones de acontecimientos o experiencias, es decir, la posibilidad de dar significados a nuestro vivir, los que, siendo significados compartidos, dan origen a narrativas que son precisamente las que hacen emerger la realidad y nuestra identidad. Esta identidad, que en muchos casos está centrada en el dolor, es la que generalmente lleva a una persona a consultar o pedir ayuda. Como plantea Anderson (1997) estamos en el 
lenguaje como un pez en el agua; por ende, es el propio lenguaje en el ejercicio terapéutico el que permite la co-construcción de distinciones, significados, narraciones, realidades e identidades ajenas al dolor; es decir, otorga la posibilidad de co-construir identidades alternativas.

Veremos a continuación de qué manera la fotografía se presenta como un elemento del lenguaje, esto es, un elemento narrativo como medio para tales cambios, no sin antes referirnos a ella brevemente.

\section{Fotografía terapéutica, fotografía social y retrato}

Sabemos que históricamente los usos de la fotografía han sido muy variados: recreativos, científicos, profesionales, culturales, artísticos, de expresión, comunicacionales, terapéuticos e, incluso, sociales. En este artículo nos detendremos particularmente en los dos últimos (la fotografía terapéutica y social), debido a su relación con la reflexión que promovemos en este escrito.

La fotografía terapéutica o fototerapia se presenta como una modalidad de intervención relativamente reciente, con desarrollo en los últimos 50 años. Aquí se utiliza la fotografía como un medio para el cambio con fines terapéuticos que promuevan la salud mental y bienestar de las personas (Viñuales, Benabarre, Alayeto, Sánchez, \& Barros-Loscertales, 2015). El uso terapéutico de la fotografía se asocia también a diferentes enfoques de abordaje, yendo desde el arte terapia —donde se precisa a la fotografía como un medio para el proceso creativo - hasta la utilización de estas para acceder a variables emocionales intrapsíquicas, traumáticas e inconscientes. Vale que aclaremos, en congruencia a la epistemología que sustenta este artículo (la que dista de los conceptos recientemente expuestos), que utilizaremos la definición más amplia de fotografía terapéutica, considerándola como un elemento de transformación en pro del bienestar de una persona. No obstante, la fotografía terapéutica también promueve el cambio social, configurándose como una herramienta flexible que cruza barreras culturales y lingüísticas, $\mathrm{y}$ que puede ser adaptada a todas las capacidades (Viñuales, 2015), relacionándose intrínsecamente con la fotografía social.

Por su parte, la fotografía social — también llamada documental o de documentalismo social-se nos presenta como una clasificación del uso de la fotografía, definida como un medio de evidencia frente a una realidad, como una forma de testimonio visual. El objetivo de la fotografía social es comunicar algo importante en función de generar un impacto en el espectador, un cambio, una transformación, una denuncia. Pretende ser un medio capaz de transmitir mensajes acerca de las preocupaciones colectivas, es decir, de las inquietudes sociales, promoviendo la generación de conciencia social y de solidaridad (Berger \& Mohr, 2008).

La modalidad particular de fotografía a la que nos referimos en nuestro artículo es el retrato. El motivo principal de este tipo de fotografía es presentar en una imagen a una o varias personas de las cuales se quiere mostrar cualidades físicas o morales. Alvarado, Matthews y Möller (2007), citando a Bourdieu (1979), señalan que el retrato se generó de la necesidad de las personas de dejar constancia de su individualidad, de poder verse a sí mismos, de saber quiénes son y cómo son, dando al espectador una imagen de sí, es decir, dejando, de una u otra manera, constancia de su identidad.

Habiendo presentado el entramado conceptual y teórico en el que se funda nuestro artículo, estamos preparados para dar respuesta a la pregunta en la que se fundamenta y centra nuestra reflexión.

\section{5. ¿De qué manera la fotografía se configura como un medio narrativo?}

No cabe duda, de acuerdo a lo que referimos en el apartado anterior respecto a la fotografía social, que esta se presenta como un medio de construcción social de realidades, siendo precisamente esta “cualidad” a la que 
pretendemos sacar partido en la presente reflexión.

Desde el construccionismo social sería iluso pensar que una fotografía es el fiel reflejo de una realidad objetiva existente ya que, como se ha establecido, nos es imposible el acceso objetivo al mundo, pudiendo acceder solo a las significaciones y relatos de nuestras experiencias, no a ellas per se. De tal manera, la fotografía se configura como una representación de una realidad - $\mathrm{o}$ de muchas posibles - que puede ser co-construida a través del lenguaje y, en consecuencia, ser cargada de un significado —o, también, de muchos posibles-.

Al referir el concepto de co-construcción, estamos dando realce al carácter colaborativo, generativo y compartido de un significado construido, siendo este, en el caso del retrato fotográfico, responsabilidad del terapeuta de la persona fotografiada (en caso de cumplir el rol de fotógrafo), así como de terceros que ven esa fotografía y emiten relatos asociados a ella. De tal forma, es de esperar que el significado que emerge y se construye de una fotografía sea uno de múltiples posibles, de acuerdo a diversas conjugaciones y elementos.

De tal manera, podemos co-construir un retrato fotográfico cargado de relatos $\mathrm{y}$, por tanto, de significados asociados a una identidad, entre muchas posibles. Esta identidad que buscamos relevar debe estar fuera de la identidad dominante propuesta por el discurso social respecto al drogadicto. Es decir, el retrato nos otorga la posibilidad de elección activa de aquello que se quiere enaltecer de una identidad a través de un significado co-construido, ya sea una característica positiva, fortaleza, pasión, hobby, habilidad, virtud, motivación, expectativa de cambio, etc. Cada uno de estos significados debe estar intrínsecamente relacionado a un relato, a una historia y a una narrativa co-construida entre la persona fotografiada y el terapeuta.

La fotografía se constituye como un medio narrativo en la medida que se configura como un elemento facilitador para la narración de otros significados y otras historias asociadas a identidades subyugadas y subalternas que no tienen posibilidad de ser contadas ni promovidas en el contexto social en el que está inserto el sujeto.

\section{De la reflexión teórica a la pragmática: una experiencia de trabajo}

El norte de este apartado es presentar, a modo de ejemplo, una de las diversas formas posibles de aplicar y materializar la reflexión teórica que hemos desarrollado en torno a la fotografía y a su práctica terapéutica en contexto del abuso de drogas.

El objetivo de la experiencia que presentaremos se centra en otorgar un espacio co-constructivo, junto a jóvenes en situación de abuso de drogas, en el que se promueva la construcción colaborativa de identidades descentradas a la identidad dominante de drogadicto; para tal transformación se utiliza como medio el retrato fotográfico, basándonos en una comprensión y abordaje epistemológico enmarcado en el construccionismo social. La experiencia se llevó a cabo en el Centro Integral de Rehabilitación (CIR) Gamma de la ciudad de Talca, Chile. El CIR Gamma es un centro de tratamiento integral de drogas de modalidad ambulatoria, gratuita y voluntaria, que ofrece diversas prestaciones biopsicosociales a jóvenes entre los 14 y 20 años de edad.

El desarrollo de la experiencia lo llevamos a cabo en varios momentos: 1) fase de propuesta de la actividad, donde exponíamos a los y las jóvenes el objetivo del proyecto en términos que puedan ser comprendidos de manera clara, invitando a la participación voluntaria. 2) Luego de contar con los participantes y con la claridad del objetivo de la actividad para ellos, se trabajó con la consigna: “¿qué me gustaría mostrar de mí en una fotografía?”, llevándolo a elementos positivos de las identidades de cada uno. Se prescindió de dar ejemplos en función de fomentar la creatividad y la participación activa de cada joven; de tal forma se evitaron influencias que pudieran limitar o restringir lo que quisieran mostrar de su identidad en un retrato. Para algunos jóvenes esto no fue fácil, por lo que se realizó una labor de exploración 
junto a ellos, siendo un facilitador de elementos identitarios que, como observadores y terapeutas, advertimos en ellos. Para otros, la consigna bastó para tener claridad de aquello que les gustaría resaltar de ellos mismos. 3) Al tener claridad de aquel o aquellos elementos a resaltar, trabajamos conjuntamente en la tarea de llevar aquel concepto o idea a una fotografía y, particularmente, a un retrato. Algunos jóvenes tenían una clara propuesta y concepto de la construcción de su fotografía, mientras otros necesitaron apoyo en esta fase. 4) Teniendo alguna noción del concepto de la fotografía, trabajado conjuntamente con todos, llevamos a cabo la fase de toma de fotografías definiendo un lugar particular de la ciudad para cada uno (generalmente asociado a algún significado personal), así como posiciones corporales, inclusión de elementos externos, enfoques fotográficos, co-construcción de metáforas y analogías, entre otros elementos constitutivos de la fotografía. 5) Elección de fotografías, títulos y relato al pie de foto. Este es uno de los pasos fundamentales, ya que establece relación con la interpretación lingüística de un relato y un título representativo de la identidad alternativa de cada joven. Este enunciado narrativo se co-construyó con ellos desde el momento en que fue capturado el retrato por el fotógrafo y fue sustentado en la propia red de significados que representan en ese instante la identidad del sujeto participante. 6) Finalmente, llevamos a cabo la exposición de fotografías en una ceremonia de finalización de año junto a todo el grupo, sus familias, otras personas significativas y algunos directivos del Senda. Expusimos a esta comunidad las fotografías impresas, junto a su título y pie de foto, en un diario mural que posteriormente se ubicó en un lugar del centro de tratamiento de manera permanente. A su vez, entregamos a cada joven su fotografía y relato al pie de foto dentro de un sobre.

A continuación, expondremos las identidades alternativas co-construidas con cada joven participante en la experiencia,

Rev.latinoam.cienc.soc.niñez juv 16 (1): 89-100, 2018 http://revistalatinoamericanaumanizales.cinde.org.co doi:10.11600/1692715x.16104 mediante la presentación de sus fotografías ${ }^{1} \mathrm{y}$ relatos alternativos.

Figura 1. Siendo yo. "La espontaneidad y alegría siempre presente en cada momento del día. La genuina conexión con el ser de uno mismo" (Dylan, 18 años).

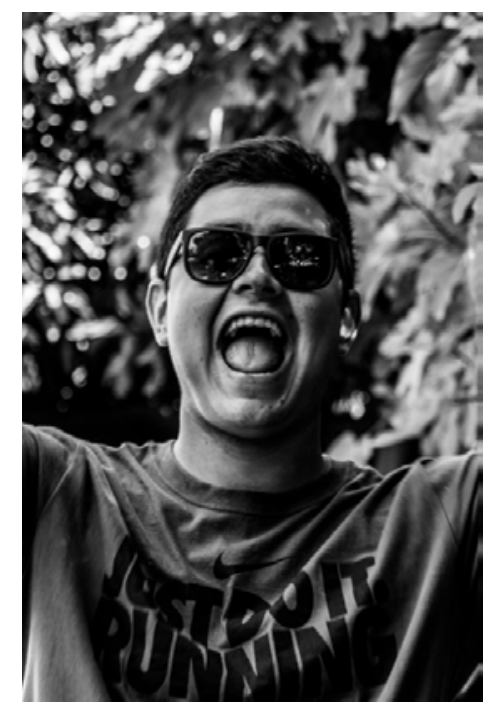

Figura 2. Rapero. "El arte de la música siempre presente como medio para la expresión de emociones. Yo aquí, freestaléandole a la vida” (Edgar, 18 años).

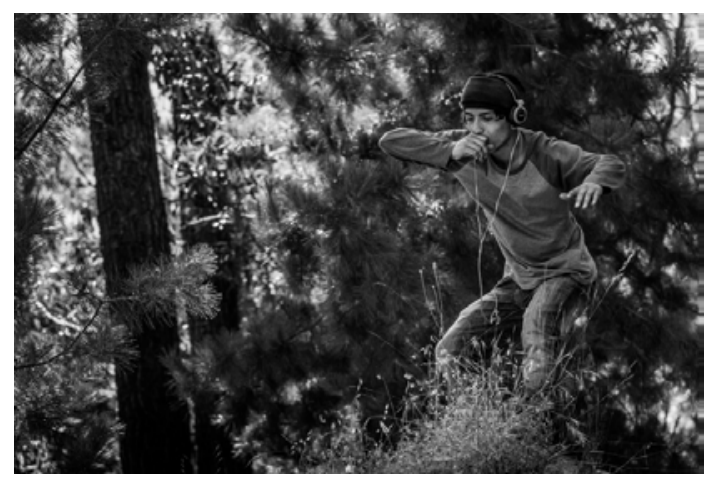

1 Todas las fotografías presentadas en este artículo fueron capturadas y elaboradas por los autores, contando con el debido consentimiento informado de los participantes para su publicación. 
Figura 3. Superación. "Sobre un árbol, como un ave esperando alzar el vuelo hacia otros cielos despejados” (Alejandro, 20 años).

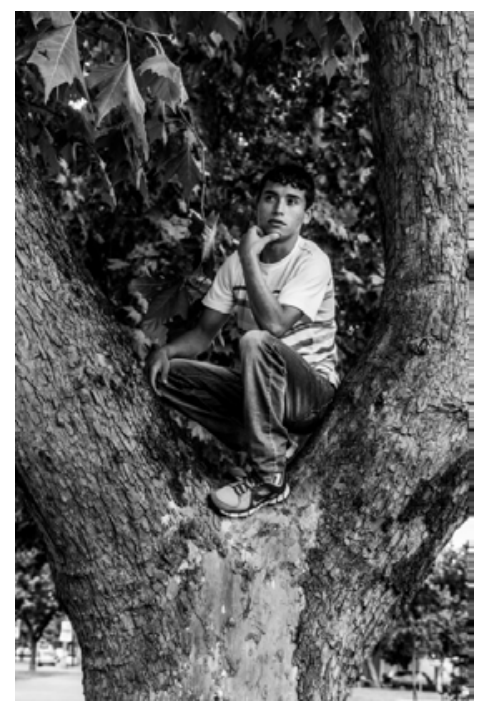

Figura 4. Creciendo juntos. "Ambos, yo y mi bebé, estamos en un proceso de cambio, ambos estamos creciendo. Mostrando lo bien que estoy ahora y los lazos que he reforzado con gente que amo” (Vale, 19 años).

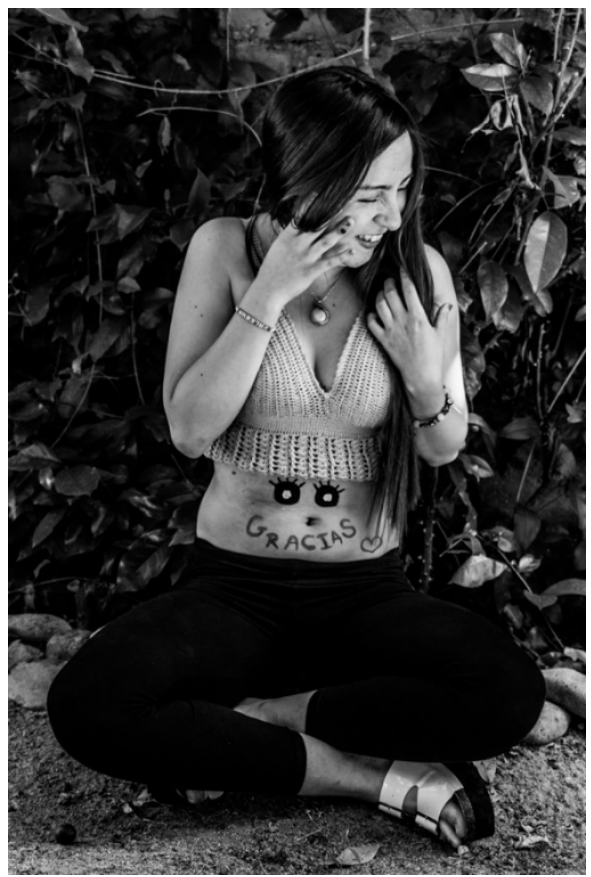

Figura 5. La fuerza. "En la cima de la montaña frente a los senderos que se han podido superar. Con los brazos abiertos esperando recorrer nuevos caminos” (Luis, 18 años).

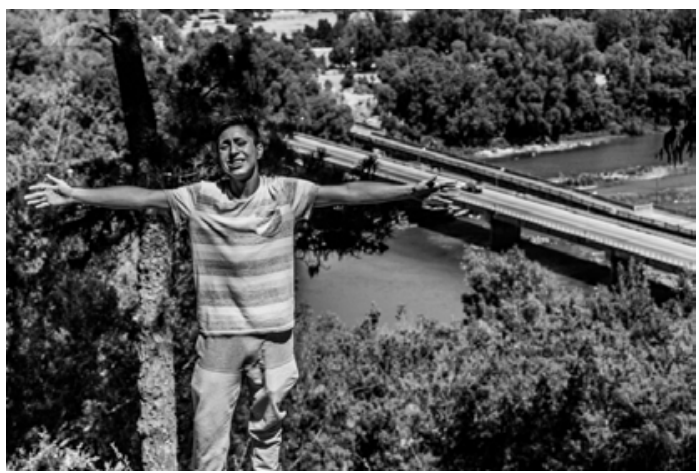

Figura 6. Solo vemos caras. "Eres capaz de mostrarte al mundo como tú quieras. Lo que se ve no es necesariamente lo que eres, porque la apariencia engaña. Vemos caras, no corazones” (Rocío, 18 años).

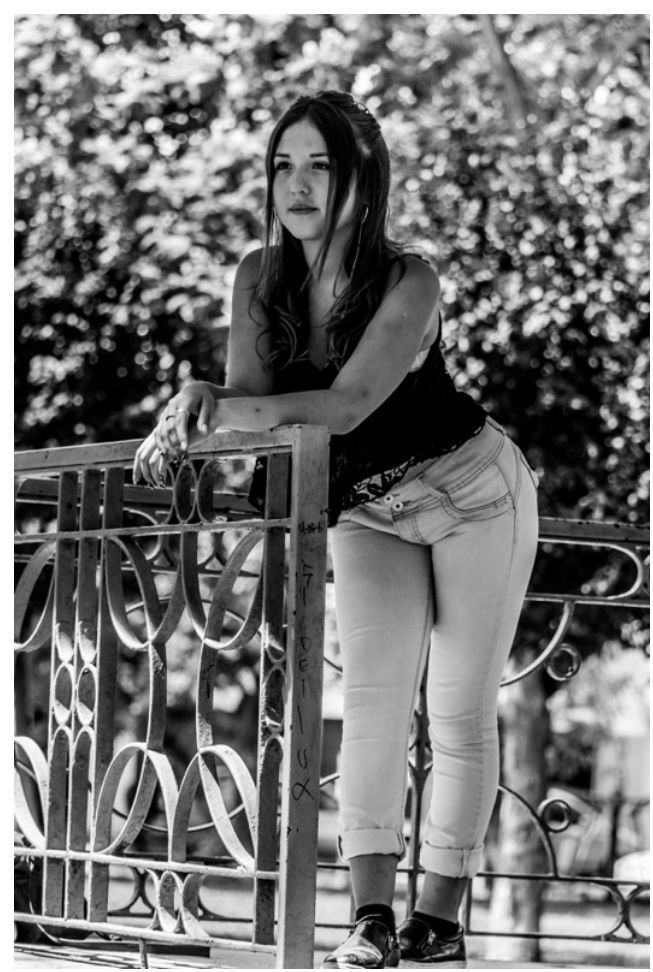


Figura 7. Trabajando en mi futuro. "El futuro comienza hoy, para el trabajo. Los sueños se cumplen despierto y despierto estoy" (Marcelo, 19 años).

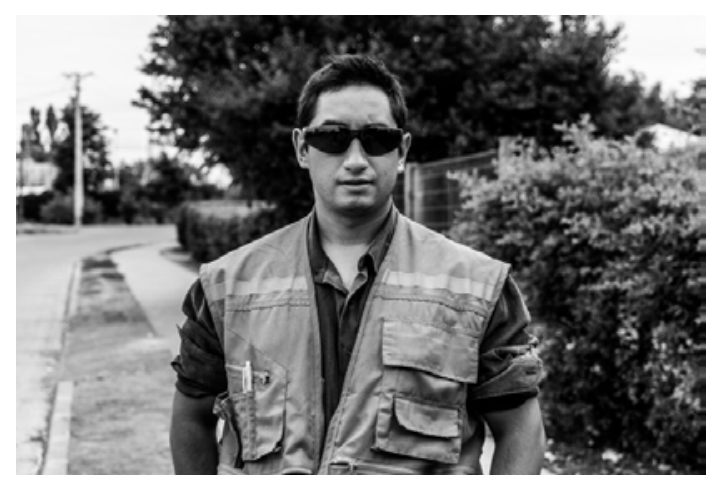

Figura 8. Peleando por los sueños. "Canalizando la energía para dar la batalla y encontrar mi camino” (Daniel, 18 años).

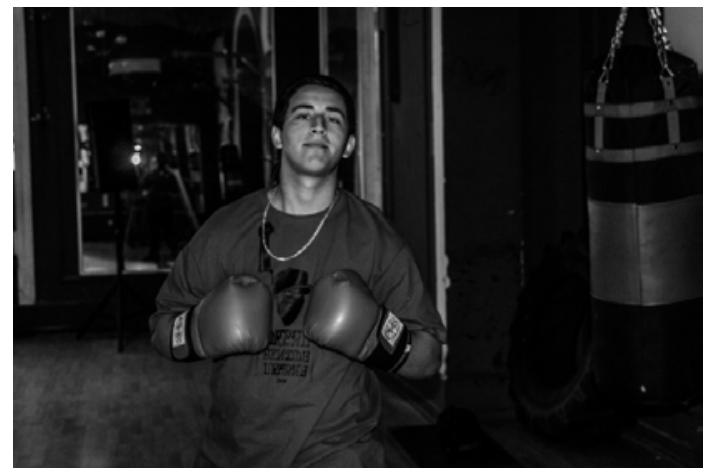

Figura 9. Mi verdad. "Mi historia en una imagen. Sentada en el presente, dándole la espalda al pasado y mirando hacia el futuro" (Pancha, 20 años).

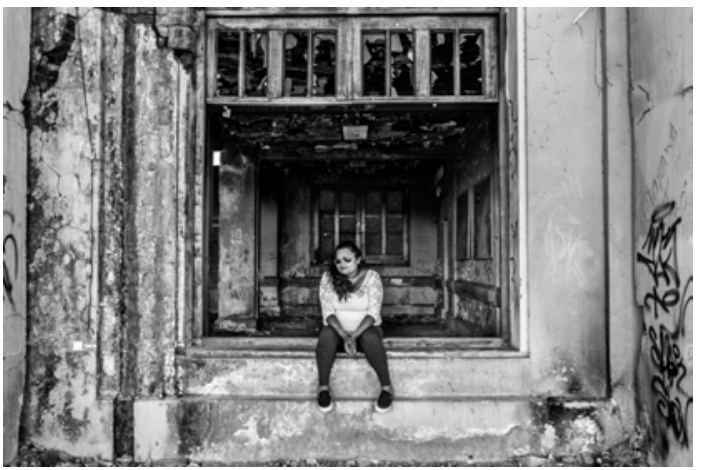

Rev.latinoam.cienc.soc.niñez juv 16 (1): 89-100, 2018 http://revistalatinoamericanaumanizales.cinde.org.co doi:10.11600/1692715x.16104
Figura 10. Randomness (aleatoriedad). "Lo que para algunos es absurdo y sin sentido, para otros abre caminos a la creatividad sin límites y expresión artística en todo su esplendor” (Tomás, 19 años).

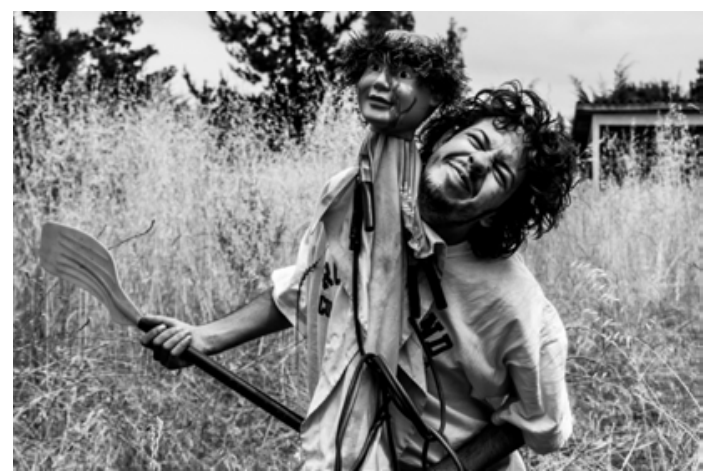

Figura 11. Vivir la vida. "Inhala el futuro, exhala el pasado. Llegó el momento de embriagarnos de sueños, amor, paciencia, felicidad, esperanza y amor” (Cata, 19 años).

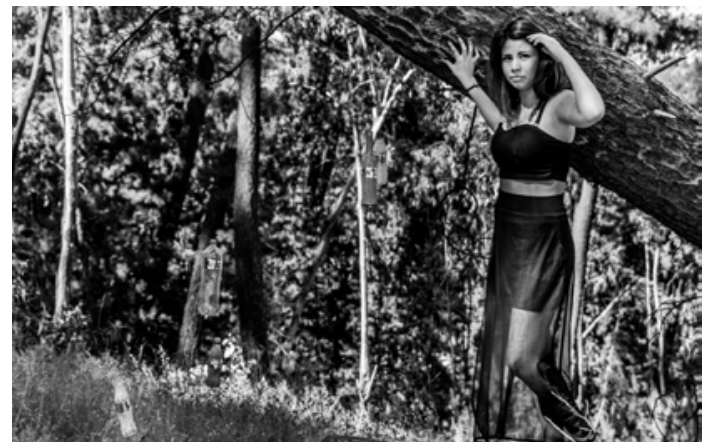

Figura 12. Seguir subiendo. "En algunas ocasiones el tronco de la vida está empinado y el camino cuesta arriba, sin embargo, seguir subiendo es mi opción” (Vannia, 19 años).

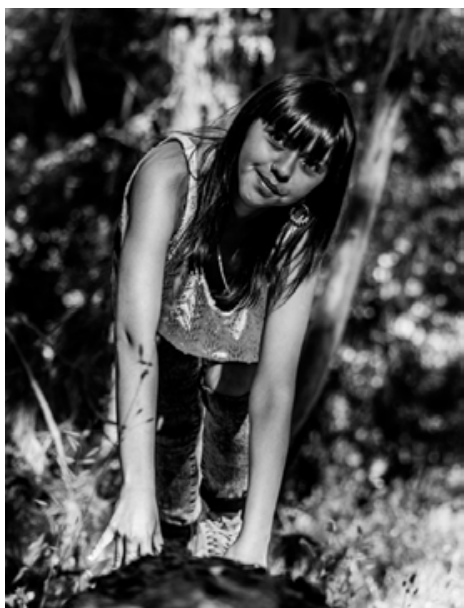




\section{Reflexiones finales}

La reflexión teórica que planteamos sobre el uso terapéutico de la fotografía como medio narrativo para la emergencia de identidades alternativas otorga la condición de posibilidad para llevar a cabo la experiencia práctica expuesta, dándole el contexto teórico que la sustenta. No obstante, la praxis nutre sin duda a la teoría y a la reflexión que desarrollamos, configurándose de tal forma en un fenómeno bidireccional y de mutua influencia, en constante apertura, dinamismo y retroalimentación. En tal escenario daremos paso a algunos comentarios finales.

Las distinciones del observador nos dicen más de este que de lo observado (Maturana, 1978). Qué distinta sería la impresión con la que el lector quedaría si hubiésemos dado importancia a los relatos construidos social y culturalmente, asociados históricamente a estos jóvenes por parte de otros observadores. Creemos que, tanto los sistemas de medios de comunicación, como el poder de las comunidades científicas, han asociado el consumo de drogas con diferentes diagnósticos y trastornos psiquiátricos como, por ejemplo, la esquizofrenia, los trastornos de la conducta alimentaria, los trastornos depresivos mayores, los trastornos antisociales, los trastornos límite de la personalidad y, por supuesto, los trastornos mentales y del comportamiento debido al consumo de sustancias. De tal manera, construyen identidades de "locos", “delincuentes", "inestables”, “impulsivos" y, como no, de drogadictos. Eso nos ha llevado a la construcción social y nominación de que todo consumidor de drogas es una persona con esas características, con una identidad fija y sin posibilidad de cambio, con el rótulo de ser un drogadicto.

Nuestra propuesta en este artículo de reflexión fue dar relevancia a otros relatos, a historias que no han tenido mucha posibilidad de ser narradas por los y las jóvenes, por sus familias e, incluso, por los mismos profesionales que pretendemos construir bienestar con ellos diariamente. El medio, la fotografía $\mathrm{y}$, particularmente, el retrato fue el facilitador de estos nuevos relatos, de estas nuevas identidades. En la experiencia práctica presentada a modo de ejemplo, fue posible dar cuenta de ello. En esta presentamos a jóvenes creativos, espontáneos, reflexivos, con sentido del humor, críticos, valientes, esforzados, solidarios, humildes, sobrevivientes, risueños, responsables, respetuosos, amables, con motivaciones claras, deportistas, apasionados y artistas, entre otros.

La exposición de las fotografías en una ceremonia final, con presencia de las familias de los y las jóvenes y directivos de Senda, no fue al azar sino, por el contrario, parte importante del proceso de co-construcción de identidades alternativas. Allí estos nuevos relatos fueron presentados a una audiencia mayor en función de que tales identidades resonaran en ella, de tal manera que otros testigos podieran ser partícipes de estos atisbos de nuevas identidades en los y las jóvenes, así como de sus nuevas redes de significados. En palabras de White (2016), esta ceremonia de definición otorga la posibilidad de que nuevas narrativas sean presentadas a una audiencia y esta se haga partícipe con la posibilidad de enriquecer los nuevos relatos. Algunas familias, directivos y personas en general participantes de la ceremonia no conocían algunas facetas de estos jóvenes como las que presentamos en este artículo. ¿Por qué?, la respuesta no es tan compleja: porque nadie habla de ellos de esa forma, porque no hay redes de conversaciones y de significado asociados a ellos con esas características, no porque no las tengan, sino que nuestras narrativas acerca de ellos, y las de ellos hacia sí mismos, priorizan otros ámbitos, no necesariamente beneficiosos, asociados a una identidad de drogadicto, limitándolos, oprimiéndolos. Por ende, quisimos romper con esa lógica y otorgar la posibilidad de co-construcción de relatos alternativos, que tributen a otras identidades que aún no han sido percibidas, concientizadas e identificadas por ellos, ni por otros, pero que están ahí, esperando ser narradas.

Dejamos las fotografías en el centro terapéutico junto a sus títulos y relatos, buscando consolidar las nuevas identidades y características asociadas a ellas, mediante la participación de terceros. Esto se concreta en la medida que todo aquel que visite el lugar, 
ya sean nuevos jóvenes o profesionales que allí se encuentren, serán partícipes de estas nuevas identidades amplificando los relatos (descripciones, definiciones y significados) respecto a las narraciones que realicen acerca de las fotografías; es decir, conocerán cualidades y no déficits respecto a estos jóvenes, moviendo el relato del foco identitario ligado al consumo de drogas. Asimismo, publicar este artículo, junto a las fotografías y relatos alternativos de los y las jóvenes, también pretende ser parte de la construcción de su identidad alternativa. ¿De qué manera?, haciendo partícipe al lector en la co-construcción de significado y de las identidades de aquellos y aquellas jóvenes. Todo aquel que lea este texto conocerá a un grupo de jóvenes asombrosos y no a un grupo de "drogadictos". De tal forma, esta publicación es parte de un rito de resonancia mayor, así como publicar los nombres reales y rostros sin ocultar viene a ser una muestra de valentía y deseo de estos jóvenes de expresar sus historias $\mathrm{y}$, por qué no, una forma de enfrentarse y luchar contra los rótulos que los marcan, discriminan y que no los representan social y culturalmente en la actualidad. Este artículo de reflexión pretende ser, en sí mismo, un testimonio de aquello, configurándose como un contradocumento, como sería llamado por White y Epston (1993), en la medida en que se convierte en un elemento de incorporación de lectores y de congregación de un público, favoreciendo la consolidación y supervivencia de nuevos significados asociados a nuevas identidades, promoviendo una revisión de los significados preexistentes - particularmente en este caso- en torno a la consideración del consumidor de drogas.

La reflexión que presentamos, y su posterior puesta en práctica, viene a promover esquemas alternativos a los esquemas de tratamiento convencional, motivando la deconstrucción de la identidad de drogadicto basada en los discursos dominantes de este como enfermo o como persona con la necesidad de ser castigada, mediante la propuesta de utilizar medios distintos a los tradicionales para la consecución de objetivos terapéuticos en el área. Con esta reflexión buscamos también presentar una invitación a la innovación.
Particularmente aquí utilizamos la fotografía; no obstante, existen innumerables medios para co-construir bienestares en personas que lo requieran, estando la música, la poesía, las artes escénicas, la danza, la pintura, las artes plásticas, etc. Todas disponibles para ser utilizadas como medios innovadores en terapia.

Por último, referimos que uno de los elementos importantes a nuestro entender es hacerse cargo de la pregunta respecto al operar epistemológico, tal como se planteó en un inicio, para lo que nos aferramos a las palabras de Demicheli (1995), quien considera que "la epistemología alude y prioriza el modo, y la serie de fenómenos que están a la base del mismo, en que los seres humanos construyen y organizan las distintas realidades en las que participan” (p. 11). En la presente reflexión la epistemología se funda en el construccionismo social y, por consecuencia, en lo que respecta a la consideración del ser humano y la construcción social de la realidad de este a través de la narración. La invitación que hacemos al lector es establecer sus propias claridades respecto a aquellas diversas formas de posicionarse, de conocer el mundo, de las premisas que subyacen su operar y, por ende, de la importancia de la consideración de los fundamentos epistemológicos en el quehacer profesional diario, en torno a los fenómenos a los que se enfrenta, estén estos en relación a las drogas o no. La consideración de tales premisas fue precisamente lo que nos otorgó la posibilidad de llevar a cabo esta experiencia, al alero de una comprensión diferente y de un abordaje consecuente con esta comprensión, del fenómeno de las drogas. Keeney (1991) refiere que si el terapeuta fuera un cocinero, su tarea estaría en dejar de prestar tanta atención a los libros de recetas e interesarse más en las teorías científicas de la nutrición; es decir, sacando el foco de la reproducción ciega y sistemática de técnicas y preocuparse más por la epistemología o los sustentos teóricos a la base de tales técnicas. Nosotros agregamos, siguiendo la analogía de Keeney, que tal giro en el pensar permitiría la preparación cooperativa de platos innovadores, atingentes y más consecuentes con las necesidades alimenticias de las personas. Entonces, esto ha de ser nuestra tarea y desafío 
en función de promover prácticas respetuosas y constructivas en torno a los fenómenos sociales a los que nos enfrentamos en la praxis del vivir.

\section{Lista de referencias}

Alvarado, M., Matthews, M., \& Möller, C. (2007). Jorge Opazo: retrato fotográfico, imagen y poder. Santiago de Chile: Pehúen.

Anderson, H. (1997). Conversación, lenguaje y posibilidades: un enfoque posmoderno de la terapia. Buenos Aires: Amorrortu.

Berger, J., \& Mohr, J. (2008). Otra manera de contar. Barcelona: Gustavo Gili.

Chacón, S. (2015). Estigma y drogas: "cuidado con los volados de la esquina". En C. Rojas (Ed.), Drogas: conceptos, miradas y experiencias (pp. 65-79). Talca: Universidad Católica del Maule.

Demicheli, G. (1995). Comunicación en terapia familiar sistémica. Santiago de Chile: Ediciones Universitarias de Valparaíso.

Gergen, K. J. (1985). The social constructionist movement in modern psychology. American Psychologist, 40, 266-275. doi:10.1037/0003-066x.40.3.266

Goolishian, H., \&Anderson, H. (2005). Narrativa y self: algunos dilemas posmodernos de la psicoterapia. En F. Schnitman (Ed.), Nuevos paradigmas, cultura y subjetividad (pp. 293-311). Buenos Aires: Paidós.

Keeney, B. (1991). Estética del cambio. Barcelona: Paidós.

Maturana, H. (1978). Biology of language: The epistemology of reality. En G. Miller, \& E. Lenneberg (Eds.), Psychology and Biology of Language and Thought (pp. 28-62). Nueva York: Academic Press.

Molina, M. F., Raimundi, M. J., \& Giménez, M. (2017). Los posibles sí mismos de los adolescentes de Buenos Aires. Revista Latinoamericana de Ciencias Sociales, Niñez y Juventud, 15(1), 455-470. doi:10.1 1600/1692715x.1512901102015

Oda, H. (2015). Drogas y enfoque de Derechos Humanos: una perspectiva crítica a los abordajes criminalizadores y patologizantes del consumo de drogas. En C. Rojas (Ed.), Drogas: conceptos, miradas y experiencias (pp. 65-79). Talca: Universidad Católica del Maule.

Rojas, C. (Ed.) (2015). Drogas: conceptos, miradas y experiencias. Talca: Universidad Católica del Maule.

Sánchez-Jiménez, M. H. (2017). Prácticas dialógicas y códigos sociolingüísticos: crisis y cambios familiares en contextos dialógicos. Revista Latinoamericana de Ciencias Sociales, Niñez y Juventud, 15(2), 1179-1190. doi:10.11600/169271 5x.1522615122016

Sluzki, C. (1996). La red social: frontera de la práctica sistémica. Barcelona: Gedisa.

Vilches, O. (2007). Construccionismo social, terapia dialógica y vínculo terapéutico. ConferenciaVIICongresoLatinoamericano de Psicoterapia y I Congreso Uruguayo de Psicoterapia, Montevideo, Uruguay.

Viñuales, D. (2015). El camino de la fotología: de las fototerapias a la fotografía. Barcelona: Autor.

Viñuales, D., Benabarre, S., Alayeto, M., Sánchez, N., \& Barros-Loscertales, A. (2015). Experiencia con fotografía terapéutica en un programa de rehabilitación psicosocial con personas con trastorno mental grave. Rehabilitación Psicosocial, 12(1), 34-38.

White, M. (2016). Mapas de la práctica narrativa. Santiago de Chile: Pranas.

White, M., \& Epston, D. (1993) Medios narrativos para fines terapéuticos. Barcelona: Paidós. 